\title{
COMPARISON OF GRADED AND UNGRADED COUSIN COMPLEXES
}

\author{
by HENRIKE PETZL and RODNEY Y. SHARP
}

(Received 24th June 1996)

\begin{abstract}
Let $R=\oplus_{n \in \mathbb{Z}} R_{n}$ be a $\mathbb{Z}$-graded commutative Noetherian ring and let $M$ be a $\mathbb{Z}$-graded $R$-module. S. Goto and $\mathrm{K}$. Watanabe introduced the graded Cousin complex ${ }^{\circ} C(M)^{\circ}$ for $M$, a complex of graded $R$-modules. Also one can ignore the grading on $M$ and construct the Cousin complex $C(M)^{\circ}$ for $M$, discussed in earlier papers by the second author. The main results in this paper are that ${ }^{\circ} C(M)^{*}$ can be considered as a subcomplex of $C(M)^{\circ}$ and that the resulting quotient complex is always exact. This sheds new light on the known facts that, when $M$ is non-zero and finitely generated, $C(M)^{\circ}$ is exact if and only if ${ }^{\circ} C(M)^{\circ}$ is (and this is the case precisely when $M$ is Cohen-Macaulay).
\end{abstract}

1991 Mathematics subject classification: 13A02, 13E05, 13D25, 13D45, $13 \mathrm{Cl} 4$.

\section{Introduction}

Let $R=\oplus_{n \in \mathbf{Z}} R_{n}$ be a $\mathbb{Z}$-graded commutative Noetherian ring, and let $M$ be a graded $R$-module. Let ${ }^{*} \operatorname{Supp}(M)$ denote the set of graded prime ideals in the support of $M$. The graded Cousin complex ${ }^{*} C(M)^{\circ}$ for $M$, introduced by $\mathrm{S}$. Goto and $\mathrm{K}$. Watanabe in [3], is a complex of graded $R$-modules and homogeneous homomorphisms having the form

$$
0 \longrightarrow M \stackrel{d^{-1}}{\longrightarrow}{ }^{*} M^{0} \stackrel{d^{0}}{\longrightarrow}{ }^{*} M^{1} \longrightarrow \ldots \longrightarrow^{*} M^{n} \stackrel{d^{n}}{\longrightarrow}{ }^{*} M^{n+1} \longrightarrow \ldots
$$

with ${ }^{*} M^{n}=\bigoplus_{\substack{p \in E^{*} \operatorname{Supp}_{M}(M) \\ \mathrm{ht}_{M}=n}}\left(\text { Coker } d^{n-2}\right)_{(\mathfrak{p})}$ for each $n \in \mathbb{N}_{0}$. (For $\mathfrak{p} \in{ }^{*} \operatorname{Supp}(M)$, the suffix "(p)" denotes homogeneous localization at p: see [2, p. 30]. Also, $\mathbb{N}_{0}$ denotes the set of non-negative integers). The homomorphisms in this complex have the following properties: for $m \in M$ and a (necessarily graded) minimal prime $\mathfrak{p}$ of $\operatorname{Supp}(M)$, the component of $d^{-1}(m)$ in $M_{(\mathrm{p})}$ is $m / 1$; for $n>0, x \in{ }^{*} M^{n-1}$ and $\mathfrak{q} \in{ }^{*} \operatorname{Supp}(M)$ with $h t_{M} q=n$, the component of $d^{n-1}(x)$ in $\left(\text { Coker } d^{n-2}\right)_{(q)}$ is $\bar{x} / 1$, where "overlines" are used to denote natural images of elements of ${ }^{*} M^{n-1}$ in Coker $d^{n-2}$. The fact that such a complex can be constructed follows from [3, Lemma 1.3.1].

It should be noted that if $L$ is a module over the commutative Noetherian ring $R^{\prime}$ and we regard $R^{\prime}$ and $L$ as trivially graded, then ${ }^{*} C(L)^{\circ}$ is (essentially) the Cousin complex $C(L)^{\circ}$ for $L$ studied in [6]. This is an algebraic analogue of the Cousin complex studied by 
Hartshorne in [4, Chapter IV]. When $L$ is non-zero and finitely generated, it is known that $L$ is Cohen-Macaulay if and only if $C(L)^{\circ}$ is exact (see [7, Theorem (2.4)]).

Again, when our graded $R$-module $M$ is non-zero and finitely generated, it is known that $M$ is Cohen-Macaulay if and only if ${ }^{*} C(M)^{\bullet}$ is exact (see [3, Theorem (1.3.3)]). Thus, for $M$ finitely generated, ${ }^{*} C(M)^{\circ}$ is exact if and only if $C(M)^{\circ}$ is exact.

This paper presents the results of a comparison of the graded and ungraded Cousin complexes for a graded module $M$ over the $\mathbb{Z}$-graded commutative Noetherian ring $R$. We show that there is a chain map $\Psi(M)=\left(\psi^{i}\right)_{i \geq-2}:{ }^{*} C(M)^{\circ} \rightarrow C(M)^{\circ}$ of complexes of $R$-modules and $R$-homomorphisms such that $\psi^{-1}: M \longrightarrow M$ is the identity map and $\psi^{i}$ is injective for all $i \in \mathbb{N}_{0}$. This will enable us to regard ${ }^{*} C(M)^{*}$ as a subcomplex of $C(M)^{\circ}$, and to form the quotient complex $Q(M)^{\circ}:=C(M)^{\circ} / \Psi(M)\left({ }^{*} C(M)^{\circ}\right)$, which we shall refer to as the degradation complex of $M$. Our main result is that this degradation complex of $M$ is always exact, so that it is automatic that ${ }^{*} C(M)^{\circ}$ is exact if and only if $C(M)^{\bullet}$ is exact!

\section{The small support}

We shall use the small support of a module over a commutative Noetherian ring, and we begin by recalling the definition of this concept.

Definition 1.1. Let $L$ be a module over the commutative Noetherian ring $R^{\prime}$. For $\mathfrak{p} \in \operatorname{Spec}\left(R^{\prime}\right)$, denote $R_{\mathfrak{p}}^{\prime} / \mathfrak{p} R_{\mathfrak{p}}^{\prime}$ by $k(\mathfrak{p})$, and, for $i \in \mathbb{N}_{0}$, let $\mu^{i}(\mathfrak{p}, L)$ (or $\left.\mu_{R^{\prime}}^{i}(\mathfrak{p}, L)\right)$ denote the $i$-th Bass number of $L$ with respect to $\mathfrak{p}$ (see [5, Theorem 18.7]).

The small support, or little support, of $L$, denoted by $\operatorname{supp}(L)$ or $\operatorname{supp}_{R^{\prime}}(L)$ (note the use of the lower case " $\mathrm{s}$ ") is defined by

$$
\begin{aligned}
\operatorname{supp}(L) & =\left\{\mathfrak{p} \in \operatorname{Spec}\left(R^{\prime}\right): \text { there exists } i \in \mathbb{N}_{0} \text { such that } \operatorname{Ext}_{R_{\mathfrak{p}}^{\prime}}^{i}\left(k(\mathfrak{p}), L_{\mathfrak{p}}\right) \neq 0\right\} \\
& =\left\{\mathfrak{p} \in \operatorname{Spec}\left(R^{\prime}\right): \text { there exists } i \in \mathbb{N}_{0} \text { such that } \mu^{i}(\mathfrak{p}, L)>0\right\} .
\end{aligned}
$$

We shall make use of the properties of the small support presented in the next lemma.

Lemma 1.2. Let $R^{\prime}$ be a commutative Noetherian ring, let $L$ be an $R^{\prime}$-module, and let $0 \longrightarrow L^{\prime} \longrightarrow M^{\prime} \rightarrow N^{\prime} \longrightarrow 0$ be an exact sequence of $R^{\prime}$-modules and $R^{\prime}$-homomorphisms. Then

(i) Ass $L \subseteq \operatorname{supp}(L) \subseteq \operatorname{Supp}(L)$;

(ii) Ass $N^{\prime} \subseteq$ Ass $M^{\prime} \cup \operatorname{supp}\left(L^{\prime}\right)$;

(iii) any one of $\operatorname{supp}\left(L^{\prime}\right), \operatorname{supp}\left(M^{\prime}\right)$ and $\operatorname{supp}\left(N^{\prime}\right)$ is contained in the union of the other two; and

(iv) if $S$ is a multiplicatively closed subset of $R^{\prime}$ and $N$ is an $S^{-1} R^{\prime}$-module, then, when $N$ is regarded as an $R^{\prime}$-module in the natural way, 


\section{COMPARISON OF GRADED AND UNGRADED COUSIN COMPLEXES 291}

$$
\operatorname{supp}_{R^{\prime}}(N)=\left\{\mathfrak{p} \in \operatorname{Spec}\left(R^{\prime}\right): \mathfrak{p} \cap S=\emptyset \text { and } \mathfrak{p} S^{-1} R^{\prime} \in \operatorname{supp}_{S^{-1} R^{\prime}}(N)\right\}
$$

Proof. (i) This is elementary.

(ii) Let $\mathfrak{p} \in$ Ass $N^{\prime}$. Then $\operatorname{Hom}_{R_{\mathrm{p}}^{\prime}}\left(k(\mathfrak{p}), N_{\mathfrak{p}}^{\prime}\right) \neq 0$. The induced exact sequence of $R_{\mathfrak{p}}^{\prime}$ modules $0 \longrightarrow L_{\mathrm{p}}^{\prime} \longrightarrow M_{\mathrm{p}}^{\prime} \longrightarrow N_{\mathrm{p}}^{\prime} \stackrel{\longrightarrow}{\longrightarrow} 0$ itself induces an exact sequence

$$
\operatorname{Hom}_{R_{\mathfrak{p}}^{\prime}}\left(k(\mathfrak{p}), M_{p}^{\prime}\right) \rightarrow \operatorname{Hom}_{R_{p}^{\prime}}\left(k(\mathfrak{p}), N_{\mathfrak{p}}^{\prime}\right) \rightarrow \operatorname{Ext}_{R_{\mathfrak{p}}^{\prime}}^{1}\left(k(\mathfrak{p}), L_{\mathfrak{p}}^{\prime}\right),
$$

from which the claim is clear.

(iii) Application of the functor $\operatorname{Hom}_{R_{\mathfrak{p}}^{\prime}}(k(\mathfrak{p}), \bullet)$ to the exact sequence

$$
0 \longrightarrow L_{p}^{\prime} \rightarrow M_{p}^{\prime} \longrightarrow N_{p}^{\prime} \longrightarrow 0
$$

produces a long exact sequence of "Ext" modules, from which the claim is clear.

(iv) Let $\mathfrak{p} \in \operatorname{Spec}\left(R^{\prime}\right)$.

If $\mathfrak{p} \cap S \neq 0$, and $s \in \mathfrak{p} \cap S$, then multiplication by $s / 1 \in R_{\mathfrak{p}}^{\prime}$ provides the zero endomorphism of $k(\mathfrak{p})$ and provides an automorphism of $N_{p}$; hence

$$
\operatorname{Ext}_{R_{p}^{\prime}}^{i}\left(k(\mathfrak{p}), N_{p}\right)=0 \quad \text { for all } i \in \mathbb{N}_{0} \text {. }
$$

If $\mathfrak{p} \cap S=\emptyset$, then $N_{\mathrm{p}}$ and $N_{\mathrm{p} S^{-1} R^{\prime}}$ are isomorphic as $R_{\mathrm{p}}^{\prime}$-modules (when $N_{\mathrm{p} S^{-1} R^{\prime}}$ is regarded as an $R_{\mathrm{p}}^{\prime}$-module by means of the natural ring isomorphism $\left.R_{p}^{\prime} \stackrel{\cong}{\longrightarrow}\left(S^{-1} R^{\prime}\right)_{p S^{-1} R^{\prime}}\right)$; it follows that, in this case,

$$
\mu_{R^{\prime}}^{i}(\mathfrak{p}, N)=\mu_{S^{-1} R^{\prime}}^{i}\left(\mathrm{p} S^{-1} R^{\prime}, N\right) \quad \text { for all } i \in \mathbb{N}_{0} .
$$

The result follows from these observations.

\section{Graded modules and local cohomology}

2.1 Notation and Terminology. Throughout the remainder of this paper, $R=\oplus_{n \in \mathbf{Z}} R_{n}$ will denote a $\mathbb{Z}$-graded commutative Noetherian ring (with identity). We shall use the notation and terminology about graded commutative Noetherian rings and graded modules over them employed by Bruns and Herzog in [2, §1.5 and \$3.6]. We shall use $\mathcal{C}(R)$ to denote the category of all $R$-modules and $R$-homomorphisms.

We shall also use many of the basic results from the above-cited sections of [2] about graded rings and graded modules without much comment.

The set of homogeneous elements of $R$ will be denoted by $h(R)$. We shall denote by * $\operatorname{Spec}(R)$ the set of graded prime ideals of $R$. For a graded $R$-module $M$, we shall denote ${ }^{*} \operatorname{Spec}(R) \cap \operatorname{Supp}(M)$ by ${ }^{*} \operatorname{Supp}(M)$.

Lemma 2.2. Let $M$ be a graded $R$-module and let $\mathfrak{p} \in{ }^{*} \operatorname{Spec}(R)$. Let $0 \neq m \in M$ (we 
are not assuming that $m$ is a homogeneous element).

(i) The ideal $\sqrt{ }(0: m)$ is graded.

(ii) If $(0: m)^{*} \subseteq \mathfrak{p}$, then $(0: m) \subseteq \mathfrak{p}$. (For an ideal a of $R$, the notation $\mathfrak{a}^{*}$ stands for the ideal of $R$ generated by all homogeneous elements of a: see [2, p. 28].)

(iii) The natural $R_{(\mathfrak{p})}$-homomorphism $\omega_{M, p}: M_{(\mathfrak{p})} \rightarrow M_{p}$, for which

$$
\omega_{M, p}(y / s)=y / s \quad \text { for all } y \in M \text { and } s \in h(R) \backslash \mathfrak{p},
$$

is injective.

Proof. (i) Each minimal prime ideal of $(0: m)$ belongs to $\operatorname{Ass}(R m) \subseteq$ Ass $M$, and every prime in Ass $M$ is graded [2, 1.5.6(b)(ii)]. Thus $\sqrt{ }(0: m)$ is an intersection of graded prime ideals.

(ii) Since $\sqrt{ }(0: m)$ is graded by (i), we can deduce, from the hypothesis that $(0: m)^{*} \subseteq \mathfrak{p}$, that $(0: m) \subseteq \sqrt{ }(0: m)=(\sqrt{ }(0: m))^{*}=\sqrt{(0: m)^{*}} \subseteq \mathfrak{p}$.

(iii) Let $y \in M$ and $s \in h(R) \backslash \mathfrak{p}$ be such that $y / s \in \operatorname{Ker} \omega_{M, p}$. Thus $(0: y) \nsubseteq \mathfrak{p}$, and so $(0: y)^{*} \nsubseteq \mathfrak{p}$ by (ii).

Lemma 2.3. Let $M$ be a graded $R$-module and let $\mathfrak{p} \in \operatorname{Supp}(M)$ with $\mathrm{ht}_{M} \mathrm{p}=n$.

(i) If $\mathfrak{p}$ is graded then there exists a chain $\mathfrak{p}_{0} \subset \mathfrak{p}_{1} \subset \ldots \subset \mathfrak{p}_{n}=\mathfrak{p}$ of graded prime ideals in $\operatorname{Supp}(M)$. (The symbol " $C$ "' is reserved to denote strict inclusion.)

(ii) If $\mathfrak{p}$ is ungraded then $\mathrm{ht}_{M} \mathfrak{p}^{*}=\mathrm{ht}_{M} \mathfrak{p}-1$.

Proof. This is proved in $[2,1.5 .8(\mathrm{~b})]$ under the additional assumption that $M$ is finitely generated. However the general case follows from this, since there exists a finitely generated graded submodule $N$ of $M$ such that $\mathfrak{p} \in \operatorname{Supp}(N)$ and $\mathrm{ht}_{M} \mathfrak{p}=\mathrm{ht}_{N} \mathfrak{p}$ : to construct such an $N$, let $\mathfrak{p}_{0} \in$ Ass $M$ be such that $\mathfrak{p}_{0} \subseteq \mathfrak{p}$ and $h t\left(\mathfrak{p} / \mathfrak{p}_{0}\right)=n$; observe that $\mathfrak{p}_{0}$ is the annihilator of a homogeneous element $m \in M$ (by $[2,1.5 .6(\mathrm{~b})(\mathrm{ii})]$ ) and let $N$ be the (necessarily graded) submodule of $M$ generated by $m$.

Lemma 2.4. Suppose that $R$ is *local (see [2, 1.5.13]) and $\mathrm{m}$ is its unique "maximal ideal. Let $L$ be a graded m-torsion $R$-module, and let $\mu_{L}: L \rightarrow L_{\mathrm{m}}$ be the natural map. Then $\operatorname{supp}\left(\operatorname{Coker} \mu_{L}\right) \subseteq\left\{\mathfrak{p} \in \operatorname{Supp}(L) \backslash{ }^{*} \operatorname{Supp}(L): \mathfrak{p}^{*}=\mathfrak{m}\right\}$.

Proof. The claim is obvious if $L=0$, and so we assume that $L \neq 0$. Now $L$ is graded, and $\mathrm{m}$-torsion; hence

$$
\operatorname{Supp}(L)=\left\{\mathfrak{p} \in \operatorname{Spec}(R): \mathfrak{p}^{*}=\mathfrak{m}\right\}=\left\{\mathfrak{p} \in \operatorname{Supp}(L): \mathfrak{p}^{*}=\mathfrak{m}\right\} .
$$

Note that $m$ is the unique minimal member of this support. Hence the Cousin complex $C(L)^{\bullet}$ for $L$ begins

$$
0 \longrightarrow L \stackrel{\mu_{L}}{\rightarrow} L_{\mathrm{m}} \longrightarrow \cdots,
$$

and, by $[6,(2.7)(i v)], \operatorname{Supp}\left(\operatorname{Coker} \mu_{L}\right) \subseteq \operatorname{Supp}(L) \backslash\{m\}$. All the claims follow. 


\section{COMPARISON OF GRADED AND UNGRADED COUSIN COMPLEXES 293}

The next lemma, which is an application of 2.2(iii), will be crucial in our comparison of graded and ungraded Cousin complexes.

Lemma 2.5. Let $M$ be a graded $R$-module, and let $q \in{ }^{*} \operatorname{Supp}(M)$. We use the $R_{(q)}{ }^{-}$ homomorphism $\omega_{M . q}$ of 2.2(iii). For each $n \in \mathbb{N}_{0}$, the homomorphism $H_{q R_{(q)}}^{n}\left(\omega_{M . q}\right)$ : $H_{\mathrm{q} R_{(q)}}^{n}\left(M_{(q)}\right) \rightarrow H_{q R_{(q)}}^{n}\left(M_{q}\right)$ is injective, and

$$
\operatorname{supp}_{R}\left(\operatorname{Coker}\left(H_{\mathfrak{q} R_{(q)}}^{n}\left(\omega_{M, q}\right)\right)\right) \subseteq\left\{\mathfrak{p} \in \operatorname{Supp}(M) \backslash{ }^{*} \operatorname{Supp}(M): \mathfrak{p}^{*}=\mathfrak{q}\right\}
$$

Proof. It is convenient to denote $(h(R) \backslash q)^{-1} X$, for an arbitrary (not necessarily graded) $R$-module $X$, by $X_{(q)}$, and to let $\omega_{X, q}: X_{(q)} \rightarrow X_{q}$ denote the natural $R_{(q)}$-homomorphism.

First note that $\Gamma_{q R_{(q)}}\left(X_{(q)}\right)=\left(\Gamma_{q}(X)\right)_{(q)}$ and $\Gamma_{q R_{(q)}}\left(X_{q}\right)=\Gamma_{q R_{q}}\left(X_{q}\right)=\left(\Gamma_{q}(X)\right)_{q}$. We can use the Flat Base Change Theorem and the Independence Theorem for local cohomology (see $[1, \$ 4.3$ and $\$ 4.2]$ ) to see that there are isomorphisms of negative strongly connected sequences of functors (from $\mathcal{C}(R)$ to $\mathcal{C}\left(R_{(\mathrm{q})}\right)$ )

$$
\Theta:=\left(\theta^{i}\right)_{i \in \mathbb{N}_{0}}:\left(H_{\mathrm{q} R_{(\mathfrak{q}}}^{i}\left((\bullet)_{(\mathfrak{q})}\right)\right)_{i \in \mathbb{N}_{0}} \stackrel{\cong}{\longrightarrow}\left(\left(H_{\mathfrak{q}}^{i}(\bullet)\right)_{(\mathfrak{q})}\right)_{i \in \mathbb{N}_{0}}
$$

and

$$
\Phi:=\left(\phi^{i}\right)_{i \in \mathbb{N}_{0}}:\left(\left(H_{q}^{i}(\bullet)\right)_{q}\right)_{i \in \mathbb{N}_{0}} \stackrel{\cong}{\longrightarrow}\left(H_{q R_{(q)}}^{i}\left((\bullet)_{q}\right)\right)_{i \in \mathbb{N}_{0}}
$$

such that $\theta^{0}$ and $\phi^{0}$ are the identity natural equivalences.

Next, observe that $\Gamma_{q R_{(q)}}\left(\omega_{X, q}\right)=\omega_{\Gamma_{q}(X), q}$ for each $R$-module $X$. It is easy to check that both

$$
\left(H_{q R_{(q)}}^{i}\left(\omega_{\bullet, q}\right)\right)_{i \in N_{0}} \quad \text { and } \quad \Phi \circ\left(\omega_{H_{q}^{i}(\bullet), q}\right)_{i \in N_{0}} \circ \Theta
$$

are homomorphisms from the connected sequence $\left(H_{q R_{(q)}}^{i}\left((\bullet)_{(q)}\right)\right)_{i \in \mathrm{N}_{0}}$ (of functors from $\mathcal{C}(R)$ to $\left.\mathcal{C}\left(R_{(q)}\right)\right)$ to. the connected sequence $\left(H_{\mathrm{q} R_{(q)}}^{i}\left((\bullet)_{q}\right)\right)_{i \in N_{0}}$ whose 0 -th constituent natural transformations are $\Gamma_{q R_{(q)}}\left(\omega_{0, q}\right)=\omega_{\Gamma_{q}(0), q}$. But there is only one such homomorphism of connected sequences. Hence

$$
H_{\mathrm{q} R_{(q)}}^{\mathrm{i}}\left(\omega_{X, q}\right)=\phi_{X}^{i} \circ \omega_{H_{q}^{i}(X), q} \circ \theta_{X}^{i}
$$

for each $R$-module $X$ and each $i \in \mathbb{N}_{0}$. Since $H_{q}^{n}(M)$ is graded, it follows from 2.2(iii) that $\omega_{H_{q}^{n}(M), q}$ is injective; hence $H_{q R_{(q)}}^{n}\left(\omega_{M, q}\right)$ is injective too. It also follows that the cokernel of this map is isomorphic to $\operatorname{Coker}\left(\omega_{H_{q}^{n}(M), q}\right)$, and consequently the final claim follows easily from Lemmas 2.4 and 1.2(iv). 


\section{The comparison results}

3.1. Properties of graded Cousin complexes (Goto and Watanabe [3]). Let $M$ be a graded module over our graded ring $R$. The graded Cousin complex ${ }^{*} C(M)^{\circ}$ for $M$ has the form

$$
0 \longrightarrow M \stackrel{d^{-1}}{\longrightarrow}{ }^{*} M^{0} \stackrel{d^{0}}{\longrightarrow}{ }^{*} M^{1} \longrightarrow \ldots \rightarrow{ }^{*} M^{n} \stackrel{d^{n}}{\longrightarrow}{ }^{*} M^{n+1} \longrightarrow \ldots
$$

and was described in the introduction. Note that it is a complex of graded $R$-modules and homogeneous homomorphisms.

We shall need the following properties of ${ }^{*} C(M)^{\circ}$.

(i) By [3, Chapter 1, §3],

${ }^{*} \operatorname{Supp}\left(\operatorname{Coker} d^{n-2}\right) \subseteq\left\{\mathfrak{p} \in{ }^{*} \operatorname{Supp}(M): h t_{M} \mathfrak{p} \geq n\right\} \quad$ for all $n \in \mathbb{N}_{0}$.

(ii) It is not difficult to deduce from (i) that, for each $n \in \mathbb{N}_{0}$ and each $\mathfrak{p} \in{ }^{*} \operatorname{Supp}(M)$ with $\mathrm{ht}_{M} \mathfrak{p}=n$, every element of $\left(\text { Coker } d^{n-2}\right)_{(\mathfrak{p})}$ is annihilated by some power of $\mathfrak{p}$.

(iii) It follows from part (i) that

$$
{ }^{*} \operatorname{Supp}\left({ }^{*} M^{n}\right) \subseteq\left\{\mathfrak{p} \in{ }^{*} \operatorname{Supp}(M): \mathrm{ht}_{M} \mathfrak{p} \geq n\right\} \quad \text { for all } n \in \mathbb{N}_{0}
$$

(iv) It is easy to deduce from [3, Lemma 1.3.1] that

$$
{ }^{*} \operatorname{Supp}\left(H^{n-1}\left({ }^{*} C(M)^{\bullet}\right)\right) \subseteq\left\{\mathfrak{p} \cdot \in{ }^{*} \operatorname{Supp}(M): \mathrm{ht}_{M} \mathfrak{p} \geq n+1\right\} \quad \text { for all } n \in \mathbb{N}_{0} .
$$

For a graded module $M$ over the graded ring $R$, we can construct both the ungraded Cousin complex $C(M)^{\bullet}$ of [6], which we shall write as

$$
0 \stackrel{e^{-2}}{\rightarrow} M \stackrel{e^{-1}}{\rightarrow} M^{0} \stackrel{e^{0}}{\rightarrow} M^{1} \longrightarrow \ldots \rightarrow M^{n} \stackrel{e^{n}}{\longrightarrow} M^{n+1} \longrightarrow \ldots
$$

and the graded Cousin complex ${ }^{*} C(M)^{\circ}$ of 3.1 . We are now going to compare these two complexes: we shall show that there is a chain map $\Psi(M)=\left(\psi^{i}\right)_{i \geq-2}$ : ${ }^{*} C(M)^{*} \longrightarrow C(M)^{\bullet}$ of complexes of $R$-modules and $R$-homomorphisms such that $\psi^{-1}: M \longrightarrow M$ is the identity map and $\psi^{i}$ is a monomorphism for all $i \in \mathbb{N}_{0}$. This will enable us to regard ${ }^{*} C(M)^{\circ}$ as a subcomplex of $C(M)^{\circ}$, and to form the quotient complex $Q(M)^{\bullet}:=C(M)^{\circ} / \Psi(M)\left({ }^{*} C(M)^{\circ}\right)$, which we shall refer to as the degradation complex of $M$.

Proposition 3.2. Let $M$ be a graded $R$-module. Use the notation of 3.1 for the graded Cousin complex ${ }^{\circ} \mathrm{C}(M)^{\circ}$ for $M$ and the notation in the above paragraph for the Cousin complex $C(M)^{\circ}$ for $M$. Use "overlines" to denote natural images of elements of terms in Cousin complexes or graded Cousin complexes in cokernels of appropriate maps in those complexes. There is a chain map 
COMPARISON OF GRADED AND UNGRADED COUSIN COMPLEXES 295

$$
\Psi(M)=\left(\psi^{i}\right)_{i \geq-2}:{ }^{*} C(M)^{\bullet} \longrightarrow C(M)^{\bullet}
$$

of complexes of R-modules and R-homomorphisms such that

(i) $\psi^{-1}: M \longrightarrow M$ is the identity map, and

(ii) for all $n \in \mathbb{N}_{0}$ and all $\left(\overline{y_{p}} / t_{\mathrm{p}}\right)_{p \in \cdot S \operatorname{Sup}(M), \mathrm{ht}_{M p=n}}{ }^{*} M^{n}$,

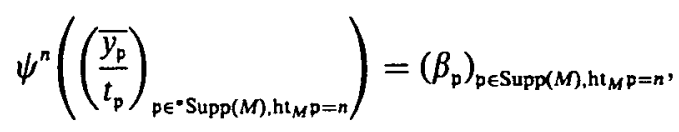

where, for $\mathfrak{p} \in \operatorname{Supp}(M)$ with $\mathrm{ht}_{M} \mathfrak{p}=n$,

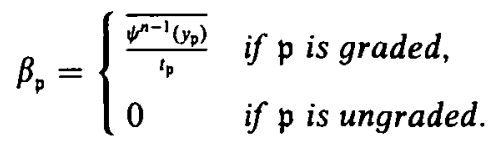

(In this notation, each $t_{p}$ denotes a homogeneous element of $R$ outside $\mathfrak{p}$, and the $y_{p}$ denote elements of ${ }^{*} M^{n-1}$; when $n=0$, interpret ${ }^{*} M^{n-1}$ as $M$ itself.) Note that

$$
\psi^{n}\left(\left(\operatorname{Coker} d^{n-2}\right)_{(\mathfrak{p})}\right) \subseteq\left(\operatorname{Coker} e^{n-2}\right)_{\mathrm{p}} \quad \text { for all } \mathfrak{p} \in{ }^{*} \operatorname{Supp}(M) \text { with } \mathrm{ht}_{M} \mathfrak{p}=n
$$

Proof. The maps $\psi^{n}\left(n \in \mathbb{N}_{0}\right)$ are constructed inductively. The key point is to show that, if $n \in \mathbb{N}_{0}$ and $y \in{ }^{*} M^{n-1}$, and $q$ is an ungraded prime of $\operatorname{Supp}(M)$ having $M$-height $n$, then the image $\bar{y}$ of $y$ in Coker $d^{n-2}$ is such that $(0: \bar{y}) \nsubseteq q$. This is achieved as follows. By 2.3(ii), $\mathrm{ht}_{M} \mathrm{q}^{*}=n-1$, and so, by 3.1(i), we have $q^{*} \notin \operatorname{Supp}\left(\operatorname{Coker} d^{n-2}\right.$ ). Hence $(0: \bar{y}) \notin q^{*}$. Therefore, by 2.2(ii), since Coker $d^{n-2}$ is a graded $R$-module, $(0: \bar{y})^{*} \nsubseteq \mathfrak{q}^{*}$, and hence $(0: \bar{y}) \notin \mathfrak{q}$.

The inductive construction of the maps $\psi^{n}\left(n \in \mathbb{N}_{0}\right)$ with the desired properties is now straightforward.

Our next major aim is to show that all the constituent homomorphisms $\psi^{n}\left(n \in \mathbb{N}_{0}\right)$ in the chain map $\Psi(M)$ of 3.2 are monomorphisms. Our method will involve a refinement of a result proved in [8]: for a non-zero module $L$ of dimension $n$ over a commutative (Noetherian) local ring $R^{\prime}$ (having unique maximal ideal $\mathfrak{m}^{\prime}$ ), it was proved in [8] that the $n$-th term in the Cousin complex $C(L)^{\circ}$ for $L$ is isomorphic to the $n$-th local cohomology module $H_{\mathrm{m}^{\prime}}^{n}(L)$.

Theorem 3.3. Let $M$ be a graded $R$-module, and let $\mathfrak{q} \in{ }^{*} \operatorname{Supp}(M)$ with $\mathrm{ht}_{M} \mathfrak{q}=n$. Use the notation of 3.1 for the graded Cousin complex ${ }^{*} C(M)^{*}$ for $M$ and the notation in the paragraph following 3.1 for the Cousin complex $C(M)^{\circ}$ for $M$. Let $\Psi(M)=\left(\psi^{i}\right)_{i \geq-2}$ : ${ }^{*} C(M)^{\circ} \rightarrow C(M)^{*}$ be the chain map of complexes of $R$-modules and $R$-homomorphisms of 3.2. Then there is a commutative diagram of $R_{(\mathfrak{q})}$-modules and $R_{(q)}$-homomorphisms 


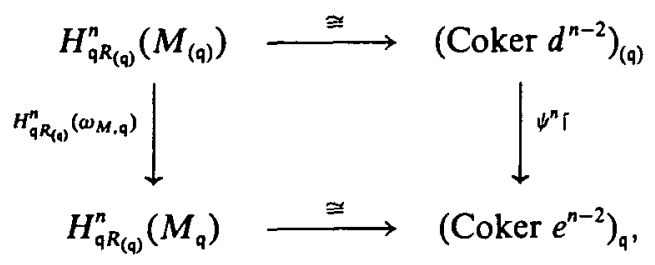

in which $\omega_{M . q}$ is as defined in 2.2(iii) and the horizontal maps are isomorphisms. Consequently, $\psi^{n}$ is a monomorphism and

$$
\operatorname{supp}_{R}\left(\left(\operatorname{Coker} e^{n-2}\right)_{q} / \psi^{n}\left(\left(\operatorname{Coker} d^{n-2}\right)_{(\mathfrak{q})}\right)\right) \subseteq\left\{\mathfrak{p} \in \operatorname{Supp}(M) \backslash * \operatorname{Supp}(M): \mathfrak{p}^{*}=\mathfrak{q}\right\}
$$

Proof. The maps $\omega_{M, q}, \omega_{M^{0}, q,}, \ldots, \omega_{M^{i}, q}, \ldots$ provide a chain map

$$
\Lambda:\left(C(M)^{\bullet}\right)_{(\mathfrak{q})} \longrightarrow\left(C(M)^{\bullet}\right)_{\mathrm{q}}
$$

of complexes of $R_{(q)}$-modules and $R_{(q)}$-homomorphisms; hence $\Lambda \circ(\Psi(M))_{(q)}$ : $\left({ }^{*} C(M)^{\circ}\right)_{(q)} \longrightarrow\left(C(M)^{\circ}\right)_{q}$ is also a chain map of such complexes.

It follows easily from 3.1 (ii) that, for each $i \in \mathbb{N}_{0}$, there is a natural $R_{(q)}$ isomorphism

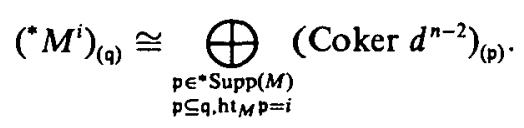

Similarly, there is a natural $R_{\mathrm{q}}$-isomorphism

$$
\left(M^{i}\right)_{q} \cong \bigoplus_{\substack{\left.p \in S_{p p p}(M) \\ p \subseteq q, h_{M}\right)}}\left(\text { Coker } e^{n-2}\right)_{p}
$$

Furthermore, when we use these two natural isomorphisms to make identifications, then the $i$-th member of the chain map $\Lambda \circ(\Psi(M))_{(q)}$ should be interpreted as the restriction $\psi^{i}\left\lceil\right.$ of $\psi^{i}$.

We conclude that there is a complex $C^{*}, R_{(\mathfrak{q})}$-isomorphic to $\left({ }^{*} C(M)^{\circ}\right)_{(\mathfrak{q})}$ and having the form

$$
0 \rightarrow M_{(q)} \stackrel{h^{-1}}{\rightarrow} C^{0} \rightarrow \ldots \rightarrow C^{i} \stackrel{h^{i}}{\rightarrow} C^{i+1} \rightarrow \ldots \rightarrow C^{n} \rightarrow 0
$$

where $C^{i}:=\bigoplus_{\substack{p \in \mathcal{S u p p}^{\circ}(M) \\ p \subseteq q_{\mathrm{h}} \mathrm{ht}_{M \mathrm{p}}=i}}\left(\text { Coker } d^{n-2}\right)_{(\mathrm{p})}$ for all $i=0, \ldots, n$, and a complex $N^{*}, R_{\mathrm{q}}$-isomorphic to $\left(C(M)^{\bullet}\right)_{q}$ and having the form 


$$
0 \rightarrow M_{q} \stackrel{g^{-1}}{\rightarrow} N^{0} \rightarrow \ldots \rightarrow N^{i} \stackrel{g^{i}}{\rightarrow} N^{i+1} \rightarrow \ldots \rightarrow N^{n} \rightarrow 0,
$$

where $N^{i}:=\bigoplus_{\substack{p \in S_{p p p}(M) \\ p \subseteq \subseteq, h M_{M} p=i}}\left(C \text { oker } e^{n-2}\right)_{p}$ for all $i=0, \ldots, n$, and a chain map $\Delta=\left(\delta^{i}\right)_{i \geq-2}$ : $C^{*} \longrightarrow N^{*}$ such that $\delta^{-1}=\omega_{M, q}$ and $\delta^{i}=\psi^{i}\left\lceil: C^{i} \longrightarrow N^{i}\right.$ for all $i=0, \ldots, n$.

The chain map $\Delta$ gives rise, for each $i=0,1, \ldots, n$, to commutative diagrams

0
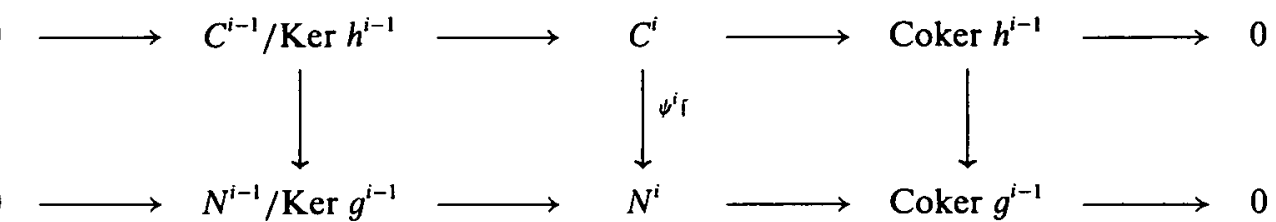

and

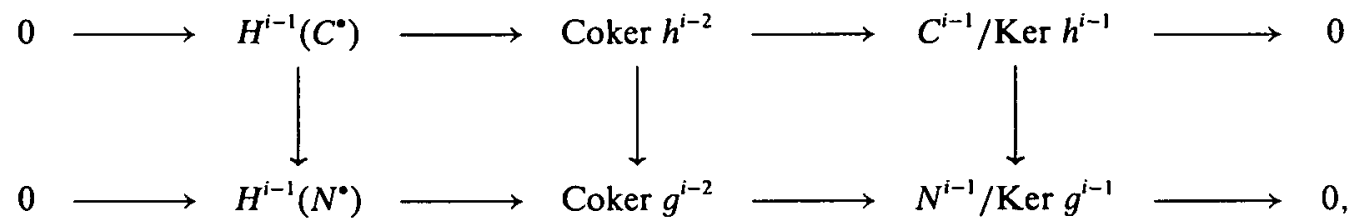

in which the vertical maps are all induced by the appropriate constituent homomorphisms of $\Delta$. (Obvious interpretations should be made when $i=0$.) Application of the $q R_{(q)}$-torsion functor $\Gamma_{q R_{(q)}}$ to each of these commutative diagrams yields a chain map of the long exact sequence of local cohomology modules arising from the top row into the long exact sequence of local cohomology modules arising from the bottom row. Our strategy is to use these chain maps in conjunction with extensions of the ideas in [8, p. 21].

Let $\mathfrak{p} \in \operatorname{Supp}(M)$ have $M$-height $h$ and be such that $\mathfrak{p} \subset \mathfrak{q}$ (so that $h<n$ ). Since there exists $r \in \mathfrak{q} \backslash \mathfrak{p}$ and every local cohomology module with respect to $q R_{(q)}$ is $q$ torsion, $H_{\left.q_{(\mathcal{Q}}\right)}^{j}\left(\left(\operatorname{Coker} e^{h-2}\right)_{p}\right)=0$ for all $j \in \mathbb{N}_{0}$. It now follows from the fact that local cohomology commutes with direct limits (see $[1, \S 3.4]$ ) that $H_{\mathrm{q} \mathrm{R}_{(\mathrm{o})}}\left(N^{i}\right)=0$ for all $i=0, \ldots, n-1$ and $j \in \mathbb{N}_{0}$. A very similar argument will show that $H_{q R_{(9)}}^{j}\left(C^{i}\right)=0$ for all $i=0, \ldots, n-1$ and $j \in \mathbb{N}_{0}$.

Let $i$ be an integer such that $0 \leq i \leq n-1$.

Let $\varepsilon=0$ or 1 . It follows from 3.1 (iv) that $\operatorname{dim}_{R_{q}}\left(H^{i-1}\left(\left({ }^{*} C(M)^{\circ}\right)_{q}\right)\right) \leq n-i-1$; therefore, by Grothendieck's Vanishing Theorem (see [1, $\left.\left.\S^{6} .1\right]\right), H_{q R_{q}}^{n-i+e}\left(H^{i-q}\left(\left({ }^{*} C(M)^{\circ}\right)_{q}\right)\right)=0$. Hence $H_{\mathrm{q} R_{(\mathrm{f})}}^{n-i+\varepsilon}\left(H^{i-1}\left(\left(^{*} C(M)^{\circ}\right)_{(q)}\right)\right)=0$ by $2.2(\mathrm{iii})$ and the Flat Base Change Theorem [1, §4.3]. Since $C^{*}$ is isomorphic to $\left({ }^{\circ} C(M)^{*}\right)_{(9)}$, it follows that

$$
H_{q R_{(Q)}}^{n-i}\left(H^{i-1}\left(C^{*}\right)\right)=H_{q R_{(q)}}^{n-i+1}\left(H^{i-1}\left(C^{*}\right)\right)=0 .
$$

Also, it follows from $\left[6,(2.7)(\right.$ vii) $]$ that $\operatorname{dim}_{R_{q}}\left(H^{i-1}\left(\left(C(M)^{\circ}\right)_{q}\right)\right) \leq n-i-1$; therefore, 
by Grothendieck's Vanishing Theorem,

$$
H_{\mathrm{q} R_{\mathrm{q}}}^{n-i}\left(H^{i-1}\left(\left(C(M)^{\bullet}\right)_{\mathrm{q}}\right)\right)=H_{\mathrm{q} R_{\mathrm{q}}}^{n-i+1}\left(H^{i-1}\left(\left(C(M)^{\bullet}\right)_{\mathrm{q}}\right)\right)=0,
$$

so that $H_{\mathrm{q} R_{q}}^{n-i}\left(H^{i-1}\left(\left(C(M)^{\bullet}\right)_{q}\right)\right)=H_{q R_{q}}^{n-i+1}\left(H^{i-1}\left(\left(C(M)^{\bullet}\right)_{q}\right)\right)=0$ by the Independence Theorem $[1, \S 4.2]$. As the complex $N^{\bullet}$ is isomorphic to $\left(C(M)^{\circ}\right)_{q}$, we have

$$
H_{\mathrm{q} R_{\mathrm{q}}}^{n-i}\left(H^{i-1}\left(N^{\bullet}\right)\right)=H_{\mathrm{q} R_{\mathrm{q}}}^{n-i+1}\left(H^{i-1}\left(N^{\bullet}\right)\right)=0 .
$$

We are now in a position to modify the argument in [8, p. 21], keeping track of commutative diagrams induced by the appropriate constituent homomorphisms of $\Delta$, to obtain a commutative diagram with the properties specified in the statement of the theorem.

The fact that $\psi^{n}$ is a monomorphism, and the final claim in the statement of the theorem, now follow from 2.5.

3.4. Definition and Notation. Let the situation be as in 3.2 . By 3.3, all the constituent homomorphisms in the chain map

$$
\Psi(M)=\left(\psi^{i}\right)_{i \geq-2}:{ }^{*} C(M)^{\bullet} \longrightarrow C(M)^{\bullet}
$$

are monomorphisms. We can therefore regard ${ }^{*} C(M)^{\bullet}$ as a subcomplex of $C(M)^{\bullet}$ : we denote the quotient complex $C(M)^{\bullet} / \operatorname{Im}(\Psi(M))$ by

$$
Q(M)^{\bullet}: 0 \rightarrow Q^{0} \stackrel{f^{0}}{\rightarrow} Q^{1} \longrightarrow \ldots \rightarrow Q^{i} \stackrel{f^{i}}{\rightarrow} Q^{i+1} \rightarrow \ldots
$$

and refer to this as the degradation complex of $M$.

Let $n \in \mathbb{N}_{0}$. Then there is an obvious isomorphism $Q^{n} \cong G^{n} \oplus U^{n}$, where

$$
G^{n}:=\bigoplus_{\substack{p \in=\text { Supp }(M) \\ \text { ht } t_{M}=n}}\left(\text { Coker } e^{n-2}\right)_{p} / \psi^{n}\left(\left(\text { Coker } d^{n-2}\right)_{(p)}\right)
$$

and

$$
U^{n}:=\bigoplus_{\substack{p \in S u p p(M) \cdot-\operatorname{Supp}(M) \\ \mathrm{ht}_{M \mathrm{p}=n}}}\left(\text { Coker } e^{n-2}\right)_{\mathrm{p}}
$$

Lemma 3.5. Let $M$ be a graded $R$-module, and let

$$
Q(M)^{\bullet}: 0 \rightarrow Q^{0} \stackrel{f^{0}}{\rightarrow} Q^{1} \rightarrow \ldots \rightarrow Q^{i} \stackrel{f^{\prime}}{\rightarrow} Q^{i+1} \longrightarrow \ldots
$$




\section{COMPARISON OF GRADED AND UNGRADED COUSIN COMPLEXES 299}

be the degradation complex of $M$, as in 3.4. Then, for all $n \in \mathbb{N}_{0}$,

$$
\operatorname{supp}\left(Q^{n}\right) \subseteq\left\{\mathfrak{p} \in \operatorname{Supp}(M) \backslash{ }^{*} \operatorname{Supp}(M): \mathrm{ht}_{M} \mathfrak{p}=n \text { or } n+1\right\}
$$

Proof. We use the notation of 3.4. Since $Q^{n} \cong G^{n} \oplus U^{n}$, it is clear that $\operatorname{supp}\left(Q^{n}\right)=\operatorname{supp}\left(G^{n}\right) \cup \operatorname{supp}\left(U^{n}\right)$. Since $k(\mathrm{p})$ is a finitely generated $R_{\mathrm{p}}$-module, the functor $\operatorname{Ext}_{R_{\mathrm{p}}}^{i}(k(\mathfrak{p}), \bullet)$ (on the category of all $R_{\mathrm{p}}$-modules and $R_{\mathrm{p}}$-homomorphisms) commutes with direct limits, for each $i \in \mathbb{N}_{0}$. It therefore follows that

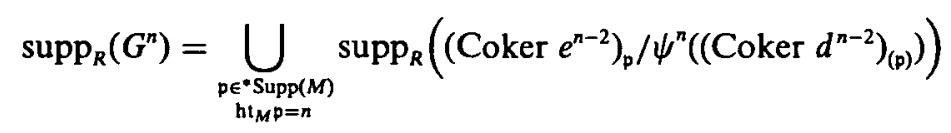

and

$$
\operatorname{supp}_{R}\left(U^{n}\right)=\bigcup_{\substack{p \in \operatorname{Supp}(M) \cdot \operatorname{Supp}(M) \\ \mathrm{h}_{M \mathcal{P}=n}}} \operatorname{supp}_{R}\left(\left(\operatorname{Coker} e^{n-2}\right)_{\mathfrak{p}}\right)
$$

The result now follows from 1.2(i),(iv) and 3.3.

Theorem 3.6. Let $M$ be a graded $R$-module. Then the degradation complex $Q(M)^{\circ}$ of $M$ is always exact.

Proof. We shall use the notation

$$
0 \rightarrow Q^{0} \stackrel{f^{0}}{\rightarrow} Q^{1} \rightarrow \ldots \rightarrow Q^{i} \stackrel{f^{i}}{\rightarrow} Q^{i+1} \longrightarrow \ldots
$$

of 3.4 for $Q(M)^{\circ}$.

The canonical sequence of complexes

$$
0 \rightarrow^{*} C(M)^{\bullet} \longrightarrow C(M)^{\bullet} \longrightarrow Q(M)^{\bullet} \longrightarrow 0
$$

induces a long exact sequence

$$
\ldots H^{i}\left({ }^{*} C(M)^{\bullet}\right) \rightarrow H^{i}\left(C(M)^{\bullet}\right) \rightarrow H^{i}\left(Q(M)^{\bullet}\right) \rightarrow H^{i+1}\left({ }^{*} C(M)^{\bullet}\right) \longrightarrow \ldots
$$

of cohomology modules, and this, together with [6, (2.7)(vii)] and 3.1(iv), shows that

$$
\begin{aligned}
\operatorname{Ass}\left(H^{i}\left(Q(M)^{\bullet}\right)\right) & \subseteq \operatorname{Supp}\left(H^{i}\left(C(M)^{\bullet}\right)\right) \cup \operatorname{Supp}\left(H^{i+1}\left({ }^{*} C(M)^{\bullet}\right)\right) \\
& \subseteq\left\{\mathfrak{p} \in \operatorname{Supp}(M): \operatorname{ht}_{M} \mathfrak{p} \geq i+2\right\} \quad \text { for all } i \in \mathbb{N}_{0}
\end{aligned}
$$

We shall make significant use of this. 
Since $H^{0}\left(Q(M)^{*}\right)$ is a submodule of $Q^{0}$, we have

$$
\begin{aligned}
\operatorname{Ass}\left(H^{0}\left(Q(M)^{\bullet}\right)\right) & \subseteq \operatorname{Ass}\left(Q^{0}\right) \subseteq \operatorname{supp}\left(Q^{0}\right) \\
& \subseteq\left\{\mathfrak{p} \in \operatorname{Supp}(M) \backslash{ }^{*} \operatorname{Supp}(M): \mathrm{ht}_{M} \mathfrak{p}=1\right\}
\end{aligned}
$$

by 3.5. (There is no ungraded prime of $M$-height 0 in $\operatorname{Supp}(M)$.) This, when used in conjunction with the immediately preceding paragraph, leads to the conclusion that $\operatorname{Ass}\left(H^{0}\left(Q(M)^{\circ}\right)\right)=\emptyset$, so that $H^{0}\left(Q(M)^{\circ}\right)=0$.

To help with an inductive argument, write $Q^{-1}:=0$, and denote the zero homomorphism from $Q^{-1}$ to $Q^{0}$ by $f^{-1}$. Note that Coker $f^{-1}=Q^{0}$, and so

$$
\operatorname{supp}\left(\operatorname{Coker} f^{-1}\right) \subseteq\left\{\mathfrak{p} \in \operatorname{Supp}(M) \backslash{ }^{*} \operatorname{Supp}(M): h_{M} \mathfrak{p}=1\right\}
$$

Now suppose that $j>0$ and that it has been shown that $H^{j-1}\left(Q(M)^{\bullet}\right)=0$ and also that each prime in the small support of $\operatorname{Coker} f^{j-2}$ lies in $\operatorname{Supp}(M)$ and has $M$-height not exceeding $j$.

The fact that $Q(M)^{\bullet}$ is exact at $Q^{j-1}$ means that there is a canonical exact sequence

$$
0 \longrightarrow \operatorname{Coker} f^{j-2} \longrightarrow Q^{j} \longrightarrow \operatorname{Coker} f^{j-1} \longrightarrow 0 .
$$

We can now use this, in conjunction with 1.2(iii), 3.5 and the inductive hypothesis, to show that

$$
\begin{aligned}
\operatorname{supp}\left(\operatorname{Coker} f^{j-1}\right) & \subseteq \operatorname{supp}\left(Q^{j}\right) \cup \operatorname{supp}\left(\operatorname{Coker} f^{j-2}\right) \\
& \subseteq\left\{\mathfrak{p} \in \operatorname{Supp}(M): \mathrm{ht}_{M} \mathfrak{p} \leq j+1\right\}
\end{aligned}
$$

Since $H^{j}\left(Q(M)^{\bullet}\right)$ is a submodule of Coker $f^{j-1}$, we have

$$
\begin{aligned}
\operatorname{Ass}\left(H^{j}\left(Q(M)^{\bullet}\right)\right) & \subseteq \operatorname{Ass}\left(\operatorname{Coker} f^{j-1}\right) \subseteq \operatorname{supp}\left(\operatorname{Coker} f^{j-1}\right) \\
& \subseteq\left\{\mathfrak{p} \in \operatorname{Supp}(M): \mathrm{ht}_{M} \mathfrak{p} \leq j+1\right\}
\end{aligned}
$$

This, when used in conjunction with the second paragraph of this proof, shows that $\operatorname{Ass}\left(H^{j}\left(Q(M)^{\bullet}\right)\right)=\emptyset$, so that $H^{j}\left(Q(M)^{\bullet}\right)=0$. This completes the inductive step.

The result of Theorem 3.6 is, of course, consistent with the results, in the case when $M$ is non-zero and finitely generated, that $M$ is Cohen-Macaulay if and only if $C(M)^{\circ}$ is exact and that $M$ is Cohen-Macaulay if and only if ${ }^{*} C(M)^{\bullet}$ is exact. However, we can say more.

Corollary 3.7. Let $M$ be a graded (not necessarily finitely generated) $R$-module, and let $i$ be an integer with $i \geq-1$.

Then $H^{i}\left({ }^{*} C(M)^{\circ}\right) \cong H^{i}\left(C(M)^{\circ}\right)$, so that, in particular, ${ }^{*} C(M)^{\bullet}$ is exact at ${ }^{*} M^{i}$ if and only if $C(M)^{*}$ is exact at $M^{i}$. Furthermore, since $H^{i}\left(^{*} C(M)^{\circ}\right)$ is equipped with a structure as 


\section{COMPARISON OF GRADED AND UNGRADED COUSIN COMPLEXES 301}

graded R-module, it follows also that the $i$-th cohomology module of the (ungraded) Cousin complex $C(M)^{\circ}$ inherits a structure as graded $R$-module. In particular, $\operatorname{Ass}_{R}\left(H^{i}\left(C(M)^{\circ}\right)\right)$ consists of homogeneous prime ideals.

Proof. This is immediate from Theorem 3.6 and the long exact sequence of cohomology modules induced by the canonical sequence of complexes

$$
0 \rightarrow{ }^{*} C(M)^{*} \rightarrow C(M)^{\bullet} \rightarrow Q(M)^{*} \rightarrow 0
$$

\section{REFERENCES}

1. M. P. BrodmanN and R. Y. Sharp, Local cohomology: an algebraic introduction with geometric applications (Cambridge University Press, 1998).

2. W. BruNs and J. Herzog, Cohen-Macaulay rings (Cambridge University Press, 1993).

3. S. Goto and K. Watanabe, On graded rings, I, J. Math. Soc. Japan 30 (1978), 179-213.

4. R. HARTSHORNe, Residues and duality (Lecture Notes in Mathematics 20, Springer, Berlin, 1966).

5. H. Matsumura, Commutative ring theory (Cambridge University Press, 1986).

6. R. Y. Sharp, The Cousin complex for a module over a commutative Noetherian ring, Math. Z. 112 (1969), 340-356.

7. R. Y. SharP, Gorenstein modules, Math. Z. 115 (1970), 117-139.

8. R. Y. ShaRP, Local cohomology and the Cousin complex for a commutative Noetherian ring, Math. Z. 153 (1977), 19-22.

Pure Mathematics Section

School of Mathematics and Statistics

UNIVERSITY OF SHEFFIELD

HICKS BUILDING

SHEFFIELD S3 7RH 